Relations industrielles

Industrial Relations

\title{
Les salaires et les prix
}

\section{Maurice Lamontagne}

Volume 5, numéro 6, mars 1950

URI : https://id.erudit.org/iderudit/1023334ar

DOI : https://doi.org/10.7202/1023334ar

Aller au sommaire du numéro

\section{Éditeur(s)}

Département des relations industrielles de l’Université Laval

ISSN

0034-379X (imprimé)

1703-8138 (numérique)

Découvrir la revue

Citer cet article

Lamontagne, M. (1950). Les salaires et les prix. Relations industrielles / Industrial Relations, 5(6), 52-54. https://doi.org/10.7202/1023334ar

Tous droits réservés @ Département des relations industrielles de l’Université Laval, 1950
Ce document est protégé par la loi sur le droit d'auteur. L'utilisation des services d'Érudit (y compris la reproduction) est assujettie à sa politique d'utilisation que vous pouvez consulter en ligne.

https://apropos.erudit.org/fr/usagers/politique-dutilisation/ 


\section{LES SALAIRES ET LES PRIX}

\section{Maurice Lamontagne}

\section{Salaires et inflation}

On a prétendu dans plusieurs milieux que l'inflation qui a suivi la période de rajustement économique d'après-guerre était attribuable dans une très large mesure à la hausse exagérée des salaires.

Toutefois, cette prétention n'est pas supportée par les faits. Pour bien comprendre ce qui s'est passé, il faut se reporter à l'année 1946 qui marque la période de transition d'une économie de guerre à une économie de paix.

A cette époque, cinq facteurs différents ont contribué à déterminer le comportement des prix. Du côté de la demande, on doit signaler la multitude de besoins insatisfaits qui s'étaient accumulés au cours du conflit, le niveau élevé du revenu des individus et des entreprises, ainsi que les vastes réserves d'épargnes liquides accumulées pendant la guerre. Grâce au volume des besoins insatisfaits et au pouvoir d'achat très élevé des consommateurs, la demande a alors atteint un niveau sans précédent.

Du côté de l'offre, le facteur principal fut la rareté relative d'une multitude de produits qui a subsisté pendant toute la période de reconversion. Cette rareté, on s'en souvient, a affecté presque tous les secteurs de l'économie.

Ces facteurs, laissés à eux-mêmes, étaient suffisants pour entraîner l'inflation. Et pourtant, ils existaient depuis le début du conflit, tandis que la montée des prix avait été relativement faible. Un dernier facteur survenu en 1946 a complètement changé la situation: la suppression des contrôles qui a entraîné le retour au marché libre.

Ainsi, la rareté relative des produits et le pouvoir d'achat élevé des consommateurs sont à l'origine de l'inflation d'après-guerre, mais la disparition des contrôles jointe au désir des producteurs d'augmenter leurs profits, en est la cause immédiate.

Une fois la hausse initiale et subite des prix survenue, les autres étapes de la période inflationnaire se sont succédé normalement. La première augmentation des prix a obligé les facteurs de production à exiger des revenus plus élevés; la hausse de la rétribution des agents productifs a contribué à gonfler les coûts de production, ce qui a servi à motiver une nouvelle augmentation des prix, laquelle entraînait un nouveau mouvement du processus inflationnaire.

Ainsi, la hausse des salaires et des coûts de production a suivi la montée des prix; elle en a été l'effet et non la cause; elle a permis au processus inflationnaire de se perpétuer, mais elle n'a pas servi à le déclencher. En fait, la seule cause immédiate de l'inflation c'est la disparition des contrôles qui a permis à la rareté relative de produits et au pouvoir d'achat élevé des consommateurs de produire leurs effets naturels.

\section{Critères de détermination du mouvement des salaires}

Tout n'est pas cependant de constater que les salaires ne sont pas à l'origine de l'inflation. On doit se demander, en plus, si les travailleurs ont reçu leur juste part de l'augmentation des prix. Pour s'en rendre compte, il faut comparer les années d'après-guerre à 1946, qui marque, comme nous l'avons déjà indiqué, le retour à une économie de paix.

Indices des taux de salaire, du coût de la vie, des prix de gros et des profits nets

$\begin{array}{ccccc}\text { Année } & \begin{array}{c}\text { Taux de } \\ \text { salaire }\end{array} & \begin{array}{c}\text { Colt de } \\ \text { 1a vie }\end{array} & \begin{array}{c}\text { Prix de } \\ \text { gros }\end{array} & \begin{array}{c}\text { Profits nets } \\ \text { après taxes }\end{array} \\ 1946 & 100.0 & 100.0 & 100.0 & 100.0 \\ 1947 & 111.6 & 110.0 & 119.0 & 135.0 \\ 1948 & 126.2 & 126.0 & 141.0 & 174.3\end{array}$

En consultant les statistiques officielles contenues dans le tableau précédent, on peut faire plusieurs constatations intéressantes.

1. Notre conclusion antérieure à savoir que les salaires n'étaient pas à l'origine de l'inflation se trouve confirmée, puisque le taux des salaires a augmenté beaucoup moins rapidement que l'indice général des prix depuis 1946.

2. Le salaire réel qui est obtenu en divisant le salaire nominal par le coût de la vie, n'a pas augmenté depuis 1946. En effet, si on divise l'indice des taux de salaire, qui atteint 126.2 en 1948 , par celui du coût de la vie, qui s'élève à 126.0 , on obtient 100.1 comme indice du salaire réel. Cela signifie que le travailleur, avec son ssalaire, ne pouvait pas se procurer plus de biens et de services en 1948 qu'en 1946.

Il faut donc reconnaître que depuis 1946 tout au moins les salaires n'ont fait que suivre les variations du coût de la vie. Cette relation s'expli- 
que facilement quand on sait que les tribunaux d'arbitrage n'acceptent généralement que la hausse du coût de la vie comme motif d'augmentation des salaires et qu'ils refusent de considérer la situation de l'entreprise, sauf lorsque celle-ci invoque son incapacité de payer.

3. En rattachant ainsi les salaires au coût de la vie, on reconnaît implicitement que la rémunération du travailleur doit correspondre au coût de sa subsistance. Mais chose étrange, en établissant cette relation, on ne s'aperçoit pas qu'on donne raison à la théorie marxiste du salaire. En effet, il y a déjà près d'un siècle, Marx affirmait qu'ä l'intérieur du système capitaliste, les salaires étaient déterminés par le coût de subsistance de l'ouvrier. Non seulement on donne ainsi raison à Marx, mais on divorce l'ouvrier de l'entreprise et on l'empêche de profiter de la situation générale de l'économie.

4. Cela ne signifie pas qu'on ne doit pas tenir compte des variations du coût de la vie dans la fixation du salaire. Le coût de la vie, tel que calculé, mesure les exigences minima ou les besoins stricts de l'ouvrier. Cependant, lorsque les salaires sont augmentés en vue de compenser une hausse du coût de la vie, il faut bien se rendre compte qu'il ne s'agit pas là d'une véritable augmentation dans la rémunération du travailleur mais bien plutôt d'un rajustement destiné à maintenir constant le salaire réel.

5. En fait, la plus élémentaire justice exige que ce que l'employé reçoit de son employeur corresponde à ce qu'il lui a donné. En d'autres termes, le salaire doit être proportionné à la valeur des services rendus. D'autre part, le principal critère qui puisse permettre d'estimer directement et équitablement la valeur des services du travail, comme des autres facteurs de production, c'est la valeur du produit. En effet, le prix d'une marchandise détermine, en définitive, la valeur des services rendus par ceux qui concourent à sa production. Ainsi la valeur du produit doit servir à fixer les salaires, comme d'ailleurs la rémunération des autres facteurs de production.

6. En consultant le tableau précédent, on peut constater la différence dans les exigences des deux principes invoqués. Il faut bien noter toutefois que la valeur de la production n'est pas représentée dans ce tableau. En effet cette valeur est obtenue en multipliant le prix moyen par les quantités produites et notre tableau ne nous renseigne que sur les prix. Cette limitation n'est cependant pas très grave puisqu'il ne s'agit pour le moment que d'illustrer le principe. Supposons donc qu'au cours de la période étudiée, la produc- tion n'a pas changé, ce qui n'est pas exact puisqu'elle a augmenté, et voyons maintenant quels résultats on obtient.

Nous avons déjà vu que depuis 1946, ce sont les variations de l'indice du coût de la vie qui ont servi à fixer les hausses de salaires. En vertu de ce principe appliqué de façon plus ou moins consciente, l'indice des taux de salaires a augmenté de $26.2 \%$, mais par contre, la valeur de la production, telle que mesurée par l'indice des prix de gros, s'est élevée de $41 \%$, ce qui a produit comme résultat une augmentation de $74.3 \%$ dans les profits nets.

$\mathrm{Si}$, au cours de la même période, on avait fixé les salaires d'après les variations dans la valeur de la production, ils auraient augmenté de $41 \%$, ce qui aurait contribué à abaisser les profits à peu près à ce niveau. Comme on peut le voir, ce système de répartition des revenus est beaucoup plus juste que le premier puisqu'il correspond à la valeur même des services rendus par chaque facteur de production.

7. De nos jours, on entend souvent revendiquer la participation des travailleurs aux bénéfces de l'entreprise. Cette réforme est excellente en soi puisqu'elle implique une démocratisation de l'économie. Au point de vue de l'augmentation des revenus du travailleur, elle représenterait une amélioration sur le mode actuel de rémunération qui ne tient compte que du coût de la vie. Toutefois, la participation aux bénéfices ne saurait donner de meilleurs résultats immédiats du point de vue du travailleur, que l'application du principe selon lequel les salaires seraient fixés d'après la valeur de la production. Et pourtant ce dernier système n'exige pas, comme le premier, une réforme de structure et il ne donne pas l'impression que dorénavant les travailleurs se partageront une partie des revenus qui, juridiquement, ne leur appartiennent pas mais appartiennent aux propriétaires de l'entreprise.

8. Lorsqu'on prétend que les mouvements des salaires devraient suivre les variations de la valeur de la production, il faut bien préciser ce qu'on entend par cette dernière expression. Dans tous les cas, il ne s'agit ni de la valeur de la production de toute l'économie, ni de celle d'une entreprise particulière, mais bien de celle de toute l'industrie. Ainsi par exemple, s'il s'agit de déterminer les salaires des employés de l'Asbestos Corporation, il faudrait tenir compte non pas des conditions générales de l'économie nationale, ni uniquement de la valeur de la production de l'Asbestos Corporation, mais de la situation générale de l'industrie de l'amiante. 
Cette façon de procéder est bien justifiée par l'extrait suivant du rapport du Service d'études de la Confédération des syndicats chrétiens de Belgique, intitulé «La Politique syndicale en matière de salaires »:

«La politique en matière de salaire devra en premier lieu se laisser guider par le gain global du secteur économique intéressé; non par le gain global de toute l'économie ni par celui de quelques entreprises de caractère aberrant. Ce qui intéresse les salariés, c'est la situation économique de leur secteur et non celle de secteurs industriels ou commerciaux qui n'ont que peu ou pas de rapports avec le leur et sur lesquels ils ne peuvent par conséquent exercer que peu ou pas d'influence... La politique des salaires doit au contraire, dans toute la mesure du possible, tenir compte de la situation moyenne ou globale du secteur. Aussi bien parmi les entreprises comme ailleurs, il y a nécessairement des retardataires; l'essentiel est que le niveau des salaires ne soit pas déterminé uniquement par les entreprises en question ».

\section{Conclusion}

De tout cet exposé, une conclusion générale se dégage. Au cours de la période de prospérité et d'inflation qui a suivi le conflit, les salaires n'ont fait que suivre la montée du coût de la vie et les travailleurs n’ont réussi quà maintenir leurs niveaux de vie. La classe ouvrière ne peut pas tolérer plus longtemps une telle situation qui l'empêche de participer comme elle y a droit au progrès économique. De telles conditions devraient également attirer l'attention des patrons, car s'ils veulent lutter efficacement contre le marxisme, ils ne doivent pas commencer d'abord par lui faire avoir raison.

Pour changer cette situation et pour respecter davantage les exigences de la justice, les suggestions suivantes semblent s'imposer:

1. L'indice du coût de la vie ne devrait servir qu'à déterminer le minimum des hausses et le maximum des baisses dans les variations des salaires. Le fait que le coût de la vie ne soit pas le seul critère de détermination du salaire ne signifie pas qu'on ne doive pas en tenir compte. Bien au contrairc. L'indice du coût de la vie mesure à chaque période les besoins de l'ouvrier, ce qu'il lui faut pour subsister. Etant donné cette signification, on conçoit facilement qu'en période de dépression, les salaires ne peuvent baisser plus rapi- dement que l'indice du coût de la vie, tandis qu'en période de prospérité, la hausse de l'indice mesure le minimum de l'augmentation de salaire à laquelle les travailleurs ont droit puisque celle-ci est juste suffisante à maintenir leurs niveaux de vie.

2. En deçà du maximum de la baisse et au delà du minimum de la hausse, tels que fixés par les variations de l'indice du coût de la vie, les salaires doivent varier selon les fluctuations de la valeur de la production de toute l'industrie concernée. Il s'agit bien de la valeur de la production, c'est-à-dire des quantités produites multipliées par le prix moyen à l'unité. Il s'agit, de plus, de la valeur de la production de toute l'industrie concernée et non pas d'une seule entreprise, qu'elle soit plus ou moins prospère que la moyenne. On aura soin, enfin, de diviser la valeur de la production par le nombre d'employés et par la période de travail, ce qui donnera, comme résultat, la productivité en valeur des ouvriers. Cette façon de procéder est la seule qui permette au travailleur d'être rémunéré selon son rendement et de participer au progrès économique, tout en tenant compte de la situation de l'entreprise et des exigences du bien commun. Cette méthode est d'ailleurs facile d'application puisque le $\mathrm{Bu}$ reau fédéral de la statistique publie chaque année des informations précises sur les principales caractéristiques de chaque secteur industriel.

3. Pour s'assurer que ces recommandations soient appliquées dans tous les cas, il faudrait que le gouvernement provincial ajoute, dans la loi, une nouvelle clause obligeant les tribunaux d'arbitrage à tenir compte de la situation de l'industrie.

Une telle clause est certainement désirable, car la plupart des tribunaux d'arbitrage, en dépit souvent de la preuve présentée, se basent uniquement sur les besoins minima de l'ouvrier ou sur le coût de la vie pour rendre leur décision et négligent systématiquement les autres éléments de la preuve.

Par ailleurs, une clause de ce genre ne serait pas injuste. Au contraire, elle obligerait tout simplement les tribunaux d'arbitrage à remplir leur devoir. En effet, ils sont chargés de déterminer le juste salaire dont la fixation ne dépend pas seulement des besoins minima de l'ouvrier mais aussi de la situation de l'industrie et des exigences du bien commun.

Enfin, cette proposition a déjà un précédent, puisque le gouvernement provincial exige depuis quelques années que les tribunaux d'arbitrage tiennent compte de la capacité de payer des services publics tels que les municipalités et les commissions scolaires. 